\title{
Relación entre marca y el consumidor en las redes sociales: estudio del vínculo afectivo de los jóvenes con dos marcas tecnológicas
}

\section{The relationship between brand and consumer in social media: study of the affective link of young people with two technological brands}

\author{
Pretel Jiménez, M., De Frutos Torres, B. y Sánchez Valle, M. ${ }^{1}$ \\ Recibido: 15-05-2018 - Aceptado: 10-07-2018 \\ DOI: https://doi.org/10.26441/RC17.2-2018-A10
}

\begin{abstract}
RESUMEN: Las emociones están presentes en la comunicación de las marcas. Sin embargo, los modelos utilizados para explicar el proceso de influencia del mensaje publicitario se han centrado en el procesamiento de la información, relegando los procesos afectivos. Este trabajo identifica los elementos que se activan en el consumidor cuando atiende a la comunicación marca y cómo este proceso contribuye a la construcción de marcas poderosas. Se plantea un estudio sobre la valoración emocional de la marca Apple comparándola con Samsung, utilizando el modelo de tres factores de Thomson, Maclnnis, y Park (2005) que mide los componentes emocionales: afecto, pasión y conexión con marcas. Los resultados muestran que la adaptación de la escala arroja índices psicométricos satisfactorios cuando es aplicado a las marcas seleccionadas. Apple se sitúa como marca mejor valorada desde el punto de vista emocional que Samsung y sus seguidores en redes sociales manifiestan mayor vínculo emocional que los que no lo son.
\end{abstract}

Palabras clave: marca; publicidad; emoción; Apple; Samsung; redes sociales.

ABSTRACT: Emotions are part of the brands communication. The models provided to explain the influence process are often focused on cognitive processing, leaving the affective process aside. The present paper identifies the elements that are activated in the consumer mind while attending brand communication and how this process supports the creation of powerful brands. The study focus on the emotional evaluation of the Apple brand compare to Samsung brand. The three-factor model proposed by Thomson, Maclnnis, y Park (2005) is used to measure the affect, passion and brand connection. The results show that the scale adaption to the Spanish sample achieves good psychometrics properties when applied to the selected brands. Apple displays better evaluation than Samsung from the emotional point of view y confirm its followers in social media have more emotional link than followersthose who are not.

Keywords: brand; advertising; emotion; Apple; Samsung; social media.

1 Marilé Pretel Jiménez es Doctora en Ciencias de la Información por la Universidad Complutense de Madrid, Master en Marketing, Comunicación e Investigación Comercial por IDEM y Profesora adjunta de Gestión de Marcas en la Universidad CEU San Pablo. mapretel.fhm@ceu.es, http://orcid.org/0000-0001-6775-047X

Belinda de Frutos Torres es Doctora en Psicología Social y Metodología de las Ciencias Sociales. Es profesora de Medios Publicitarios y Psicología de la Publicidad en la Universidad de Valladolid. belinda.defrutos@hmca. uva.es, https://orcid.org/0000-0002-9391-8835

María Sánchez Valle es Doctora en Periodismo por la Universidad Pontificia de Salamanca y Profesora adjunta de Comunicación Integrada y Comunicación Empresarial e Institucional en la Universidad CEU San Pablo. mvaIle.fhum@ceu.es. https://orcid.org/0000-0003-1497-2938 


\section{Introducción}

El cambio profundo del entorno actual definido por una saturación de mensajes publicitarios, la multiplicación de canales y la ausencia de diferenciación de los productos que pertenecen a una misma categoría (Benavides, 2014) ha generado que las marcas exploren nuevos territorios para crear un vínculo con su público objetivo. Entre estas nuevas vías de acceso al consumidor se encuentran las estrategias dirigidas a crear un vínculo emocional y convertir a la marca en una lovemark (Roberts, 2005). Roberts (2005) considera que cuando una marca construye la relación con sus consumidores basándose en el cumplimiento de sus promesas, innovando y creando valor, en el respeto, en la búsqueda de la simplicidad, cuidando su reputación, siendo íntegra, aceptando la responsabilidad de ofrecer calidad para crear confianza se convierte en una marca amada.

La relevancia de las marcas en la sociedad actual es un hecho incontestable. Vivimos rodeados de marcas que nos ayudan a definirnos y a relacionarnos con los demás. El consumidor toma decisiones de compra y crea vínculos con las marcas, al mismo tiempo que las marcas tratan de generar una comunicación persuasiva. En este artículo se ahonda en el proceso de construcción de la marca verbalizado en el discurso publicitario considerando que los públicos a los que se dirige la comunicación evalúan esos discursos de forma favorable despertando sentimientos positivos que, a su vez, se traduzcan en una experiencia positiva hacia la marca (Zeitlin y Westwood, 1989 y Gutiérrez, 2002).

Estas relaciones emocionales entre la marca y el consumidor han sido estudiadas entre otros autores por Strong (1925), Lavidge y Steiner (1961), Krugman (1965), Petty y Cacioppo (1986), Barry y Howard (1990) Vaktratsas y Amber (1999) tratando de encontrar cuáles eran los elementos emocionales que unen a las marcas con sus consumidores.

El mismo investigador resume su propio modelo en la siguiente cita:

The paper compares the persuasion (strong) and reinforcement (weak) theories of advertising and uses neuroscience as the base for a third, MAC (memory dominates affect which in turns dominates cognition), model of what advertising does for brands (Ambler, 2001: p. 299).

Dentro del rol que juegan las emociones en la publicidad es posible identificar distintas clases de estrategias. Zeitlin y Westwood (1986) plantean que las emociones pueden jugar tres papeles distintos dentro de la comunicación en el ámbito del marketing que son contribuir a comunicar atributos de los productos, actuar como beneficio en sí mismo e influir directamente en las actitudes.

Los dos primeros aspectos explican la capacidad de la publicidad como una herramienta para generar en el consumidor una respuesta de carácter cognitivo y, por tanto, incrementar la notoriedad del anuncio y de la marca. Además de su poder para provocar una respuesta afectiva, donde los sentimientos originados por la publicidad se trasladan a la marca (Gutiérrez, 2002; Zeitlin y Westwood, 1986).

El aumento del uso de elementos emocionales y experienciales en la actividad publicitaria de las marcas ha dado lugar a su estudio por parte de algunos autores tales como Arnold (1992), Roberts (2005), López 
Vázquez (2007) o Martín Requero (2007), que ven en el fenómeno un alejamiento de los argumentos basados en las características intrínsecas del producto para basar su discurso en los valores de la marca y en la experiencia que genera.

Antonio Núñez, director de planificación estratégica de la agencia de publicidad SCPF, observa que

La atención de los consumidores no se capta mediante argumentos racionales, sino a través de imágenes que emocionen. Cuanto más intensa sea esta emoción, más profunda será la conexión neurológica conseguida en el cerebro del consumidor en potencia, por lo que las campañas de publicidad deben reforzar estas redes neuronales, ya que son las que finalmente motivan la compra impulsiva de determinados productos (Vilaseca, 2007).

Entre todos estos planteamientos teóricos, se considera en este estudio que es el modelo formulado por Thompson, MacInnis y Park (2005) y denominado Modelo de tres factores el que da una mejor respuesta a la necesidad de conocer el apego de marca construido a través de atributos emocionales. Los autores realizaron un estudio psicométrico a 120 estudiantes donde se les exponía a una escala (consistente, fiable y validada) que reflejaba los apegos emocionales de estos consumidores a las marcas. Para ello, los investigadores siguieron los procesos recomendados por Churchill (1979) en donde se dejaban ver los atributos dominantes expresados por los consumidores y que definían mejor el brand attachment ${ }^{2}$. El méto- do seguido proponía una escala basada en tres dimensiones emocionales (afecto, pasión y conexión) que revelaban la fuerza del apego de la marca al consumidor, y demostraba la fiabilidad de la escala por encima de las hasta entonces realizadas, teniendo a su vez una validez predictiva ya que arrojaba resultados sobre lealtad de marca y la voluntad de comprar/invertir en ella mientras que las escalas hasta ese momento aplicadas solo mostraban parámetros de actitudes favorables, satisfacción e involucración con la marca.

Son varias las aportaciones teóricas sobre el papel de la emoción en la comunicación de las marcas, aunque se haya puesto en relación con aspectos como la notoriedad de marca o el comportamiento de compra, uno de los elementos definitorios de una lovemark se concreta en la relación marca-consumidor.

La relación con las marcas ha cambiado en la medida en que el espacio de comunicación marca- consumidor se ha desplazado hacia los medios interactivos. En este nuevo espacio de relación son los propios usuarios los que interactúan e inician el contacto con las marcas. Y es precisamente con algunas de esas marcas con las que se sienten identificados o ligados desde una perspectiva no solo racional sino también emocional. En este estudio se tiene en cuenta como territorio prioritario la relación de los jóvenes con las marcas a través de la redes sociales puesto que ya en 2013 (Cocktail Analysis, 2012) la penetración de las redes sociales es de un 97\% entre los jóvenes. Tendencia que se ha mantenido a lo largo de los años. Estudios

\footnotetext{
2 El término Brand attachment está referido a la conexión emocional entre personas y marcas.
} 
más actuales indican que la penetración de usuarios de las redes sociales se mantiene estable desde 2011, siendo en 2016 del 91\% (Cocktail Analysis, 2016).

Apoyó esta decisión el dato de que el $54 \%$ de los internautas entre 21 y 24 años y el $55 \%$ de los de 25 a 34 opinaban que las redes sociales son un buen sitio para aprender más sobre las marcas. Entre las motivaciones de los usuarios para seguir a las marcas se encuentra la necesidad de interacción con la marca. Así el 48\% se unen a la marca por su pasión hacia la misma y señalan que su identificación con la marca es importante para ellos (41\%) (TNS, 2012). Teniendo en cuenta estos datos, parece lógico pensar que las marcas que forman parte del universo de las denominadas lovemarks serán más seguidas por el público joven.

\section{Planteamiento y metodología}

El objetivo principal de este estudio es conocer el vínculo emocional que los jóvenes establecen con la marca Apple. Un estudio preliminar realizado por las autoras de este artículo puso de manifiesto que Apple constituía una marca de referencia en el universo de los adolescentes y jóvenes y puede constituir una lovemark. La elección de la marca Apple para la realización de este estudio también se justifica porque claramente la marca mantiene un discurso publicitario de contenido aspiracional, por ello se plantea su estudio desde las tres dimensiones emocionales propuestas por Thomson, MacInnis y Park (2005) de modo que, cobra trascendencia conocer los atributos emocionales asociados con la marca Apple y ponerlos en relación con la interacción de los consumidores con la marca. Estos atributos pueden estar presentes en mayor o menor grado, tanto en seguidores como en no seguidores de la marca. Además para enriquecer los resultados y entender mejor el caso de éxito de esta marca, se analiza también a su mayor competidor Samsung desde la perspectiva emocional, para comprobar cuál de estas dos marcas tiene mayor conexión emocional con los sujetos objeto de estudio.

Ya en 2012 el 54\% de los internautas entre 21 y 24 años y el $55 \%$ de los de 25 a 34 opinaban que las redes sociales son un buen sitio para aprender más sobre las marcas. Entre las motivaciones de los usuarios para seguir a las marcas se encontraban la necesidad de interacción con la marca. Así el 48\% se unen a la marca por su pasión hacia la misma y señalan que su identificación con la marca es importante para ellos (41\%) (TNS, 2012). Esta tendencia continua en 2016 cuando la autenticidad y naturalidad son valores que ganan importancia en las redes sociales y se manifiesta una tendencia a compartir datos con las marcas aunque a cambio de una mayor exigencia en su comunicación. Mientras 8 de cada 10 usuarios consideran las redes sociales un buen lugar para interactuar con las marcas (Cocktail Analysis, 2016). Desde la perspectiva de las empresas, la presencia de las marcas en redes sociales es cada vez más importante. La IAB (2017) pone de manifiesto que se ha pasado de un escenario en el que la marcas no conocían las redes sociales a que la práctica totalidad estén presentes en ellas. Aspectos como la humanización de las marcas y conseguir un mayor nivel de interacción adquieren una gran importancia.

\subsection{Objetivos e hipótesis}

Teniendo en consideración todo lo expuesto con anterioridad se formulan los siguientes objetivos específicos: 
Objetivo 1. Conocer el alcance de los fans ${ }^{3}$ de la marca Apple en el universo de las marcas de tecnología y telefonía entre los jóvenes.

Objetivo 2. Identificar si la marca Apple muestra mayor vínculo emocional que su rival Samsung

Objetivo 3. Evaluar si existen diferencias en la evaluación afectiva entre fans y no fans de la marca Apple.

Objetivo 4. Comparar el vínculo emocional de Apple y Samsung entre seguidores y no seguidores de ambas marcas.

La hipótesis de partida es que la marca Apple genera mayor vínculo emocional que la marca Samsung tanto entre jóvenes seguidores como no seguidores. Ese vínculo emocional será mayor en los jóvenes declarados como fans de la marca Apple que los que no son fans. Así mismo se espera consistencia entre el comportamiento de ser fan de una marca y la evaluación afectiva hacia dicha marca. De modo que los seguidores de la marca Samsung manifestarán mayor vínculo emocional hacia su marca que los no seguidores.

\subsection{Muestra}

Para probar esta hipótesis se aplicó una encuesta a 641 alumnos procedentes de diversas Universidades de España, de carácter público y privado. Las edades de los participantes estaban entre los 21 y los 25 años. La forma de extracción de la muestra fue con el método bola de nieve. El punto de partida fueron estudiantes universitarios de la Universidad CEU San Pablo (Facultad de Humanidades) y de la Universidad de Valladolid (Facultad de Ciencias Sociales,
Jurídicas y de la Comunicación). La recogida de datos se realizó en los meses de marzo a mayo de 2014.

\subsection{Descripción del cuestionario}

Para llevar a cabo el estudio cuantitativo se elaboró un cuestionario auto-cumplimentado en el que se recogen las variables sociodemográficas sobre edad, sexo, ocupación principal y si eran seguidores de marcas en redes sociales y en caso afirmativo se recogía el número de marca de las que eran fans.

Para identificar a los seguidores de marcas en redes sociales se plantea una pregunta abierta solicitando el nombre de las marcas a las que siguen en las redes sociales en cuatro sectores: bebidas, telefonía móvil, ropa/complementos y electrónica. Las respuestas fueron codificadas a fin de identificar a los seguidores de la marca Apple y de la marca Samsung.

Para la construcción de la escala de atributos emocionales se utilizó el Modelo de tres Factores propuesto por Thomson, MacInnis, y Park (2005) en cuya investigación aplicaron la escala a tres marcas, entre ellas Apple. La escala propuesta se agrupa en tres componentes emocionales, ligados a la conexión con la marca: afecto (affection) descrita por las emociones: afectuosa, querida, amistosa y pacífica. Pasión (passion) descrita por las emociones: apasionado, encantado, cautivado. Conectado (connection) descrita por las emociones: conectado, unido, apegado.

Para el desarrollo de la escala se procedió a traducir los adjetivos utilizados en la versión original de Thomson, MacInnis, y Park (2005) generándose una lista de sinónimos.

3 Un fan es aquel usuario que con su perfil en redes sociales se hace seguidor del perfil de una marca comercial. 
La elección de los adjetivos se realizó tomando como referencia los usos lingüísticos más utilizados en Español. Para el componente de afecto los adjetivos emocionales utilizados fueron: compañera, sensible, cariñosa, simpática, cordial y conmovedora. Para el componente de pasión: apasionada, excitante, cautivadora, emocionante, deseada y encantadora. Y por último, para el componente de conexión: me identifico, conecto, me define, me siento unido, me engancha.

La lista de adjetivos de cada factor se presentaba de forma desordenada. En primer lugar, los participantes realizaban la evaluación de la marca Apple y posteriormente evaluaban los mismos atributos para Samsung. El orden de presentación de los atributos se fue rotando de forma aleatoria para que la posición no influyera en su evaluación. Cada adjetivo estaba enmarcado en la pregunta: ¿en qué medida consideras Apple como una marca...(adjetivo)? y ¿en qué medida consideras a Samsung como una marca...(adjetivo)?

Las respuestas se recogieron en una escala de 4 puntos que representaban el grado de identificación del adjetivo con la marca.
Los valores fluctuaban entre 1 (nada), 2 (poco), 3 (algo) y 4 (mucho). Para calcular la puntuación global en cada factor se han sumado las puntuaciones obtenidas en cada atributo. Previamente se han obtenido las propiedades psicométricas de los tres factores de la escala. Los resultados muestran que los coeficientes Alpha de Cronbach de las tres dimensiones son muy elevados, situándose entre 0,95 y 0,86 (Tabla 1), indicativo de la consistencia del rasgo evaluado. $\mathrm{Al}$ mismo tiempo se llevó a cabo un análisis factorial por mínimos cuadrados ponderados para comprobar si el listado de atributos se ajustaba a la estructura tridimensional. Los resultados muestran una estructura de tres factores que dan cuenta del $61,8 \%$ de la varianza explicada. Las puntuaciones de los atributos se corresponden con las dimensiones para las que habían sido propuestos al aplicar una rotación de los factores por el método varimax. Tan sólo el adjetivo "encantadora" tiene una puntuación mayor en la dimensión de afecto que en la dimensión pasión para el que había sido propuesto. En el resto de los casos las puntuaciones en el factor están entre 0,80 y 0,48 (Tabla 2)

Tabla1. Coeficientes de fiabilidad de las tres dimensiones de la escala de atributos emocionales

\begin{tabular}{|l|c|c|c|}
\hline & $\begin{array}{c}\text { Alfa de } \\
\text { Cronbach }\end{array}$ & $\begin{array}{c}\text { Alfa de Cronbach basada en } \\
\text { los elementos tipificados }\end{array}$ & $\begin{array}{c}\text { № de } \\
\text { elementos }\end{array}$ \\
\hline Conexión Apple &, 946 &, 946 & 5 \\
\hline Pasión Apple &, 862 &, 861 & 6 \\
\hline Afecto Apple &, 866 &, 868 & 6 \\
\hline
\end{tabular}

Fuente: Elaboración propia 
Tabla 2. Matriz de puntuaciones Análisis Factorial Método Máxima Verosimilitud Rotación Varimax

\begin{tabular}{|l|l|l|l|}
\hline $\begin{array}{c}\text { En qué medida consideras } \\
\text { Apple una marca }\end{array}$ & Factor $\mathbf{1}$ & Factor $\mathbf{2}$ & Factor $\mathbf{3}$ \\
\hline que me define & $\mathbf{0 , 8 1 2}$ & 0,329 & 0,242 \\
\hline con la que me identifico & $\mathbf{0 , 8 0 4}$ & 0,323 & 0,257 \\
\hline me siento unido & $\mathbf{0 , 8 0 3}$ & 0,376 & \\
\hline me siento enganchado & $\mathbf{0 , 7 6 4}$ & 0,232 & 0,229 \\
\hline con la que conecto & $\mathbf{0 , 7 6 3}$ & 0,268 & 0,338 \\
\hline encantadora & 0,28 & 0,674 & $\mathbf{0 , 3 6 4}$ \\
\hline cariñosa & 0,392 & $\mathbf{0 , 6 4 8}$ & 0,202 \\
\hline conmovedora & 0,325 & $\mathbf{0 , 6 2 4}$ & 0,294 \\
\hline cordial & 0,281 & $\mathbf{0 , 6 2 3}$ & 0,335 \\
\hline sensible & 0,268 & $\mathbf{0 , 5 9 4}$ & \\
\hline simpática & 0,226 & $\mathbf{0 , 5 5 3}$ & 0,373 \\
\hline compañera & 0,461 & $\mathbf{0 , 4 8 3}$ & 0,245 \\
\hline emocionante & 0,261 & 0,389 & $\mathbf{0 , 6 5 7}$ \\
\hline cautivadora & 0,219 & 0,254 & $\mathbf{0 , 6 4 3}$ \\
\hline excitante & 0,216 & 0,341 & 0,631 \\
\hline deseada & 0,204 & & 0,615 \\
\hline apasionada & & 0,452 & $\mathbf{0 , 5 3 2}$ \\
\hline
\end{tabular}

Fuente: Elaboración propia

\section{Resultados}

\subsection{Descriptivos de la muestra}

La muestra formada por 641 encuestados se sitúan en un rango de edad entre los 18 y los 25 años, siendo la edad media de 21,5 años (Tabla 3).

El $31 \%$ son hombres y el $69 \%$ son mujeres. El mayor porcentaje de los participantes está estudiando (73,5\%), el 14,7\% combina estudios y trabajo y el 8,3\% sólo trabaja.

El seguimiento de las marcas en las redes sociales es un fenómeno bastante generalizado entre los participantes del estudio. El $87,7 \%$ siguen a alguna marca en las redes.

Los datos de la encuesta nos permiten cuantificar el número de marcas que estos jóvenes siguen en la Red, es decir, como se les denomina en el entorno interactivo aquellos que son fans. El número máximo de marcas de las que se declaran seguidores se eleva hasta 20. El gráfico 1 muestra la distribución de frecuencias del número de marcas a las que siguen. En el tramo inferior de la gráfica se aprecia que el 26,8\% sigue entre una y tres marcas. Uno de cada cuatro seguidores declara haberse vinculado a 4 marcas; otro $25,7 \%$ afirma seguir entre 5 y 7 marcas. En el extremo opuesto destaca el 12,8\% que declara seguir a más de 20 marcas.

En un análisis más detallado de los resultados se puede apreciar las diferencias entre los seguidores de una sola marca y los que siguen a más de 20. Según se aprecia en el gráfico 1 el 42,4\% de la muestra sigue entre 3 y 6 marcas, y el 25,1\% el porcentaje más alto de seguidores de entre 3 y 4 marcas. Los internautas más activos son fan de más de 20 marcas, este grupo representa el 8,1\% de la 
muestra. En el otro extremo se encuentran los seguidores de una o dos marcas, que son el $12,8 \%$. Este resultado pone de manifiesto que la actividad en las redes sociales está bas- tante generalizada entre los jóvenes y se sigue con intensidad teniendo en cuenta el elevado número de marcas con las que mantienen una interacción voluntaria.

Tabla 3. Rango de edades de la muestra en frecuencia y en porcentaje

\begin{tabular}{|c|c|}
\hline Edad & Porcentaje \\
\hline 18 & 2 \\
\hline 19 & 4,2 \\
\hline 20 & 22,9 \\
\hline 21 & 24 \\
\hline 22 & 17,2 \\
\hline 23 & 15,6 \\
\hline 24 & 8,9 \\
\hline 25 & 5,1 \\
\hline Total & 100 \\
\hline
\end{tabular}

Fuente: Elaboración propia

Gráfico 1. Porcentaje de número de marcas comerciales que los jóvenes siguen en redes sociales.

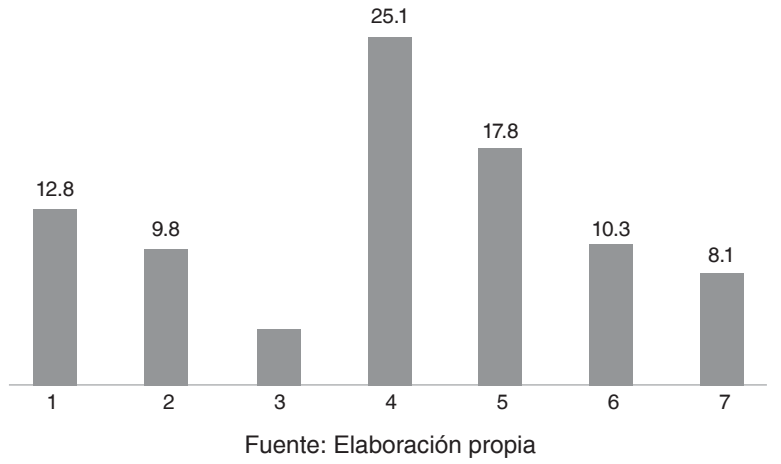

3.2. Resultados descriptivos del estudio cuantitativo

El objetivo principal del estudio empírico es identificar el mapa cognitivo asociado a la marca Apple e identificar los aspectos afectivos que la diferencian y la definen frente a la marca Samsung.

El componente emocional en el deseo del consumidor joven de contactar con las marcas se analiza con los 17 descriptores del vínculo emocional. La tabla 4 muestra los descriptivos obtenidos para cada atributo en la marca Apple cuyas puntuaciones medias en los atributos fluctúan entre 2,1 y 3,5 puntos. Teniendo en cuenta que por encima de los dos puntos la escala refleja la 
identificación con el atributo en cuestión se comprueba que hay vínculo emotivo con la marca Apple. Las mayores puntuaciones se obtienen en los atributos "excitante", "cautivadora", "emocionante" y "deseada" (Tabla 4).
La comparación por sexo en todos los atributos de la escala no arrojó diferencias estadísticamente significativas, por lo tanto, la evaluación de los participantes del vínculo emocional hacia la marca Apple no viene influida por el género.

Tabla 4. Estadístico descriptivo en cada uno de los adjetivos emocionales sobre la marca Apple

\begin{tabular}{|l|c|c|c|}
\hline \multirow{2}{*}{$\begin{array}{l}\text { En qué medida consideras } \\
\text { Apple como una marca }\end{array}$} & \multicolumn{2}{|c|}{ Media } & Desv. típ. \\
\cline { 2 - 4 } & Estadístico & Error típico & Estadístico \\
\hline compañera & 2,51 &, 041 & 1,044 \\
\hline sensible & 2,43 &, 040 & 1,005 \\
\hline cariñosa & 2,08 &, 037 &, 926 \\
\hline simpática & 2,73 &, 036 &, 915 \\
\hline cordial & 2,64 &, 035 &, 888 \\
\hline conmovedora & 2,34 &, 041 & 1,028 \\
\hline apasionada & 2,96 &, 039 &, 983 \\
\hline excitante & 3,03 &, 037 &, 936 \\
\hline cautivadora & 3,21 &, 038 &, 966 \\
\hline emocionante & 3,03 &, 037 &, 936 \\
\hline deseada & 3,54 &, 032 &, 819 \\
\hline encantadora & 2,59 &, 038 &, 964 \\
\hline con la que me identifico & 2,37 &, 042 & 1,063 \\
\hline con la que conecto & 2,64 &, 042 & 1,066 \\
\hline que me define & 2,30 &, 041 & 1,031 \\
\hline me siento unido & 2,22 &, 042 & 1,074 \\
\hline me siento enganchado & 2,25 &, 045 & 1,127 \\
\hline
\end{tabular}

Fuente: Elaboración propia

\section{3. Análisis de los resultados en función} de los objetivos e hipótesis planteados

3. 3. 1. Objetivo 1. Conocer el alcance de los fans de la marca Apple en el universo de las marcas de tecnología y telefonía entre los jóvenes

Del total de 641 entrevistados declaran en espontáneo (es decir, sin mencionar ninguna marca) 197 nombran a Apple como marca de la que se han hecho fan (Gráfico 2).
3.3.2. Objetivo 2. Identificar si la marca Apple muestra mayor vínculo emocional que su rival Samsung

A continuación, en coherencia con el siguiente objetivo del trabajo, se analiza si los jóvenes sienten mayor vínculo emocional con la marca Apple que son su rival Samsung. En concreto, se utilizan las puntuaciones de los tres factores que configuran la emoción según el modelo de Thomson, MacInnis, y 
Park (2005) descrito en el apartado de metodología y se lleva una comparación de medias (estadístico t de Student) en cada una de las dimensiones emocionales: afecto, pasión y conexión. De este modo se podrá conocer cuáles son las dimensiones emocionales con los que más identifican una marca frente a la otra para el total de la muestra.

Gráfico 2. Alcance de la marca Apple en redes sociales

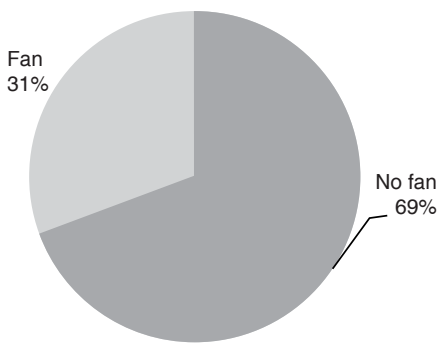

Fuente: Elaboración propia

Según se aprecia en el gráfico 3, la valoración media de Apple es superior a la de Samsung en las tres dimensiones emocionales. En la dimensión "afecto" Apple obtiene una media de 2,5 puntos frente a 2,3 de Samsung. Una vez realizado el contraste estadístico se comprueba que la diferencia de medias es estadísticamente significativa $(\mathrm{t}=5,058 ;$ g.l. $=640 ;$ sig. $=0,000)$. La segunda dimensión "pasión" refleja mayores diferencias siendo de 3,1 para Apple frente al 2,5 Samsung; en este caso la diferencia también es estadísticamente significativa $(\mathrm{t}=17,811$; g.l.=640; sig. =0,000). La dimensión "conexión" obtiene una media de 2,4 puntos para Apple y 2,1 puntos para Samsung; la diferencia en la valoración de ambas marcas es estadísticamente significativa ( $\mathrm{t}=3,874$; g.l. $=640$; sig. =0,000). Por lo tanto se puede afirmar que los participantes en el estudio manifiestan mayor vínculo afectivo con la marca Apple que con la marca Samsung en las tres dimensiones evaluadas, siendo la pasión la que más diferencia establece entre ambas.

Gráfico 3. Valoración media de las marcas Apple y Samsung

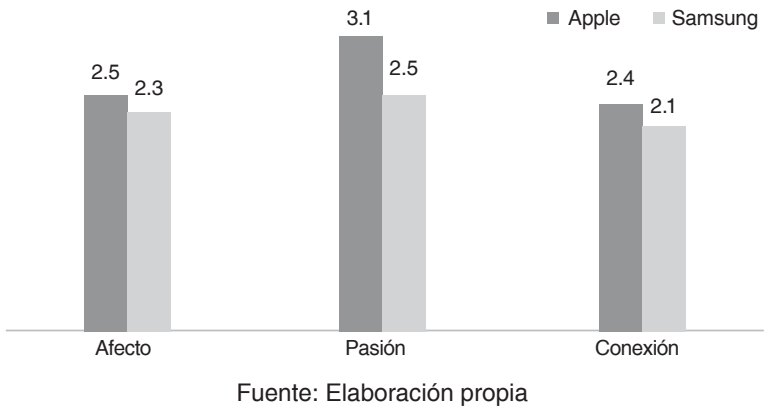


3. 3. 3. Objetivo 3. Evaluar si existen diferencias entre fans y no fans de la marca Apple

Para la concreción del tercer objetivo se utilizan de nuevo las puntuaciones en las tres dimensiones de la emoción de la marca Apple comparando entre fans y no fans de la marca. Según se ha hipotetizado, los seguidores de la marca en las redes sociales mostrarán mayor vínculo emocional que los no seguidores.

Gráfico 4. Valoración emocional de la marca Apple en redes sociales

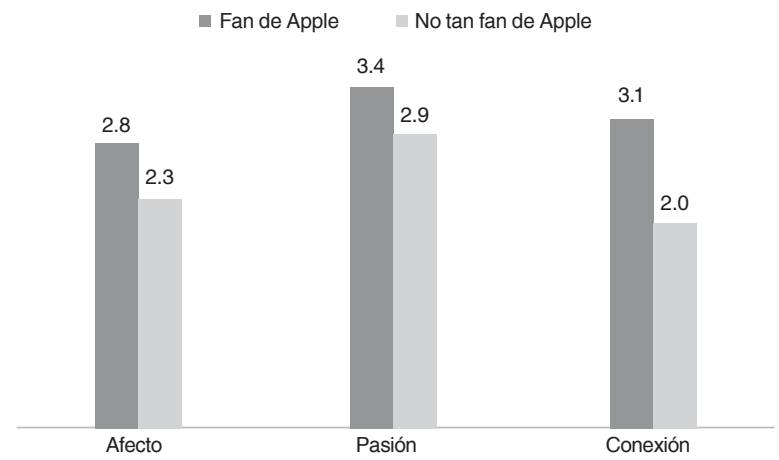

Fuente: Elaboración propia

En el gráfico 4 se muestran los resultados obtenidos en las tres dimensiones donde se aprecia que la valoración de los fans es superior a los no fans de la marca. Para comprobar si las diferencias son estadísticamente significativas se efectúa un análisis de varianza de un solo factor (ANOVA). En la dimensión de afecto los fan de Apple obtienen una puntuación media de 2,8 frente a 2,3 de los no fan, siendo esta diferencia estadísticamente significativa $(\mathrm{F}=85,671$; g.l. = 639; sig.0,000). La dimensión pasión es la mejor valorada tanto por ambos grupos siendo las diferencias entre fan y no fan de nuevo estadísticamente significativas $(\mathrm{F}=67,673$; g.l. = 639; sig.0,000). En la dimensión conexión se pone de manifiesto de una forma más clara las diferencias en la valoración entre el grupo de fans y no fans. En el grupo de fan la puntuación media es de 3,1 frente a 2,0 de los no fan, como cabe esperar estas diferencias son estadísticamente significativas. $(\mathrm{F}=204,365 ;$ g.l. $=639$; sig.0,000). Por lo tanto se confirma la hipótesis planteada acerca del mayor vínculo emocional de los seguidores en el afecto, la pasión y la conexión que sienten con la marca.

\section{3. 4. Objetivo 4. Comparar el vínculo emocional de Apple y Samsung entre se- guidores y no seguidores de ambas marcas.}

Finalmente, se pone a prueba la consistencia del vínculo emocional diferenciado hacia cada marca. Para ello se divide la muestra en cuatro grupos según se identifica como: fan de Apple, fan de Samsung, fan de ambas marcas y no fans. Mediante un análisis de varianza (ANOVA) se compara la evaluación de las tres dimensiones emocionales de la marca Apple y de la marca Samsung entre los cuatro grupos (Tabla 5). 
Tabla 5. Anova de la evaluación de las dos marcas en los cuatro grupos de fans

\begin{tabular}{|l|r|r|r|}
\hline & \multicolumn{1}{|c|}{ F } & gl & Sig. \\
\hline Afecto en Apple & 32,552 & 3 &, 000 \\
\hline Pasión en Apple & 24,168 & 3 &, 000 \\
\hline Conexión en Apple & 70,237 & 3 &, 000 \\
\hline Afecto en Samsung & 24,418 & 3 &, 000 \\
\hline Pasión en Samsung & 23,001 & 3 &, 000 \\
\hline Conexión en Samsung & 49,612 & 3 &, 000 \\
\hline
\end{tabular}

Fuente: Elaboración propia

Los resultados muestran que las diferencias son estadísticamente significativas en las tres dimensiones: afecto, conexión y pasión para las dos marcas. A continuación se analiza dónde se están dando esas diferencias. Para este fin se muestran las puntuaciones medias de los cuatro grupos en cada dimensión.

En coherencia con hipótesis planteada en la dimensión del afecto hacia Apple los fans de Apple obtienen una puntuación media superior que los fans de Samsung, que los que no son fan de ninguna de las dos marcas, y también de los que son fans de ambas marcas. En las tres comparaciones las diferencias son estadísticamente significativas (Tabla 6). En la evaluación de la pasión hacia la marca Apple de nuevo la puntuación de los fans es significativamente superior a los que no son fans de ninguna de las dos marcas y superior a los fans de la competidora Samsung; no obstante, las diferencias no alcanzan la significación cuando se compara con el grupo de participantes que se declara fan de ambas marcas (Tabla 6). En cuanto a la conexión con la marca Apple, se confirma que hay diferencias estadísticamente significativas en la comparación del grupo de fans con los grupos restantes (Tabla 6).

En cuanto a las puntuaciones de la marca Samsung se aprecia que los fans de la marca tienen valoraciones más elevadas en las tres dimensiones de la emoción que el resto de los grupos (Tabla 7). La comparación entre el grupo de seguidores de Samsung y el resto de los grupos muestra que en la dimensión del afecto las diferencias son estadísticamente significativas con el grupo de fans de Apple, con el grupo que no es fan de Apple, ni de Samsung; no obstante no llegan a alcanzar la significación con los que se declaran fans de ambas marcas. En la evaluación de la pasión hacia Samsung los resultados apuntan en el mismo sentido, los seguidores de la marca tienen puntuaciones superiores a los fans de Apple y los que no son fans de Apple, ni de Samsung siendo estas diferencias estadísticamente significativas. Al igual que en la comparación anterior, no hay diferencia entre los fans de Samsung y los fans de Apple y Samsung. En la evaluación de la conexión de nuevo se confirma la superioridad en la evaluación de los seguidores de Samsung sobre los otros dos grupos. 
Tabla 6. Puntuaciones medias de la evaluación de Apple en los cuatro grupos

\begin{tabular}{|l|r|r|r|r|r|r|}
\hline & N & Media & $\begin{array}{c}\text { Desviación } \\
\text { estándar }\end{array}$ & $\begin{array}{c}\text { Error } \\
\text { estándar }\end{array}$ & $\begin{array}{c}\text { Diferencia de } \\
\text { medias (1) }\end{array}$ & Sig. \\
\hline Evalúa afecto en Apple & & & & & & \\
\hline Fan de Apple & 161 & 2,8965 & 0,6982 & 0,05503 & & \\
\hline No fan de Apple, ni Samsung & 321 & 2,2347 & 0,70264 & 0,03922 & 0,6618 & 0,000 \\
\hline Fan de Samsung & 123 & 2,4024 & 0,68848 & 0,06208 & 0,49404 & 0,000 \\
\hline Fan de Samsung y Apple & 36 & 2,588 & 0,73408 & 0,12235 & 0,30852 & 0,017 \\
\hline Total & 641 & 2,4529 & 0,75063 & 0,02965 & & \\
\hline & & & & & & \\
\hline Evalúa pasión en Apple & & & & & & \\
\hline Fan de Apple & 161 & 3,4234 & 0,51673 & 0,04072 & & \\
\hline No fan de Apple, ni Samsung & 321 & 2,8775 & 0,73044 & 0,04077 & 0,54593 & 0,000 \\
\hline Fan de Samsung & 123 & 3,000 & 0,75295 & 0,06789 & 0,42340 & 0,000 \\
\hline Fan de Samsung y Apple & 36 & 3,2593 & 0,64543 & 0,10757 & 0,16414 & 0,193 \\
\hline Total & 641 & 3,0595 & 0,71919 & 0,02841 & & \\
\hline & & & & & & \\
\hline Evalúa conexión en Apple & & & & & & \\
\hline Fan de Apple & 161 & 3,1391 & 0,77202 & 0,06084 & & \\
\hline No fan de Apple, ni Samsung & 321 & 2,0262 & 0,8842 & 0,04935 & 1,11296 & 0,000 \\
\hline Fan de Samsung & 123 & 2,0699 & 0,83607 & 0,07539 & 1,06921 & 0,000 \\
\hline Fan de Samsung y Apple & 36 & 2,7889 & 0,82938 & 0,13823 & 0,35024 & 0,025 \\
\hline Total & 641 & 2,3569 & 0,97257 & 0,03841 & & \\
\hline
\end{tabular}

Fuente: Elaboración propia

Tabla 7. Puntuaciones medias de la evaluación de Samsung en los cuatro grupos

\begin{tabular}{|l|r|r|r|r|r|r|}
\hline Evalúa afecto en Samsung & N & Media & $\begin{array}{c}\text { Desviación } \\
\text { estándar }\end{array}$ & $\begin{array}{c}\text { Error } \\
\text { estándar }\end{array}$ & $\begin{array}{c}\text { Diferencia de } \\
\text { medias (2) }\end{array}$ & Sig. \\
\hline Fan de Samsung & 123 & 2,7046 & 0,74101 & 0,06681 & & \\
\hline Fan de Apple & 161 & 2,1605 & 0,67466 & 0,05317 & 0,54415 & 0,000 \\
\hline No fan de Apple, ni Samsung & 321 & 2,1511 & 0,66505 & 0,03712 & 0,55352 & 0,000 \\
\hline Fan de Samsung y Apple & 36 & 2,6481 & 0,7557 & 0,12595 & 0,05646 & 0,665 \\
\hline Total & 641 & 2,2876 & 0,72456 & 0,02862 & & \\
\hline & & & & & & \\
\hline Evalúa pasión en Samsung & & & & & & \\
\hline Fan de Samsung & 123 & 2,8835 & 0,72615 & 0,06547 & & \\
\hline
\end{tabular}




\begin{tabular}{|l|r|r|r|r|r|r|}
\hline Fan de Apple & 161 & 2,2805 & 0,69707 & 0,05494 & 0,6023 & 0,000 \\
\hline No fan de Apple, ni Samsung & 321 & 2,3505 & 0,73407 & 0,04097 & 0,53300 & 0,000 \\
\hline Fan de Samsung y Apple & 36 & 2,838 & 0,75539 & 0,1259 & 0,04551 & 0,740 \\
\hline Total & 641 & 2,4626 & 0,76108 & 0,03006 & & \\
\hline & & & & & & \\
\hline Evalúa conexión en Samsung & & & & & & \\
\hline Fan de Samsung & 123 & 2,8407 & 0,88149 & 0,07948 & & \\
\hline Fan de Apple & 161 & 1,8224 & 0,75299 & 0,05934 & 1,01829 & 0,000 \\
\hline No fan de Apple, ni Samsung & 321 & 1,9645 & 0,86175 & 0,0481 & 0,87616 & 0,000 \\
\hline Fan de Samsung y Apple & 36 & 2,8667 & 0,89952 & 0,14992 & $-0,02602$ & 0,871 \\
\hline Total & 641 & 2,1476 & 0,93287 & 0,03685 & & \\
\hline
\end{tabular}

\section{Fuente: Elaboración propia}

En la comparativa de ambas marcas vemos como el fan de Apple está significativamente más conectado a su marca que el fan de la marca Samsung. También es de destacar que los que no son fans se sienten más conectados con la marca Apple que con Samsung. Podría por tanto deducirse que el apego a la marca Apple se consigue de una manera satisfactoria entre aquellos que no se declaran fan de ninguna marca (Gráfico 6).

Gráfico 5. Valoración de las tres dimensiones de la marca Apple para los cuatro grupos

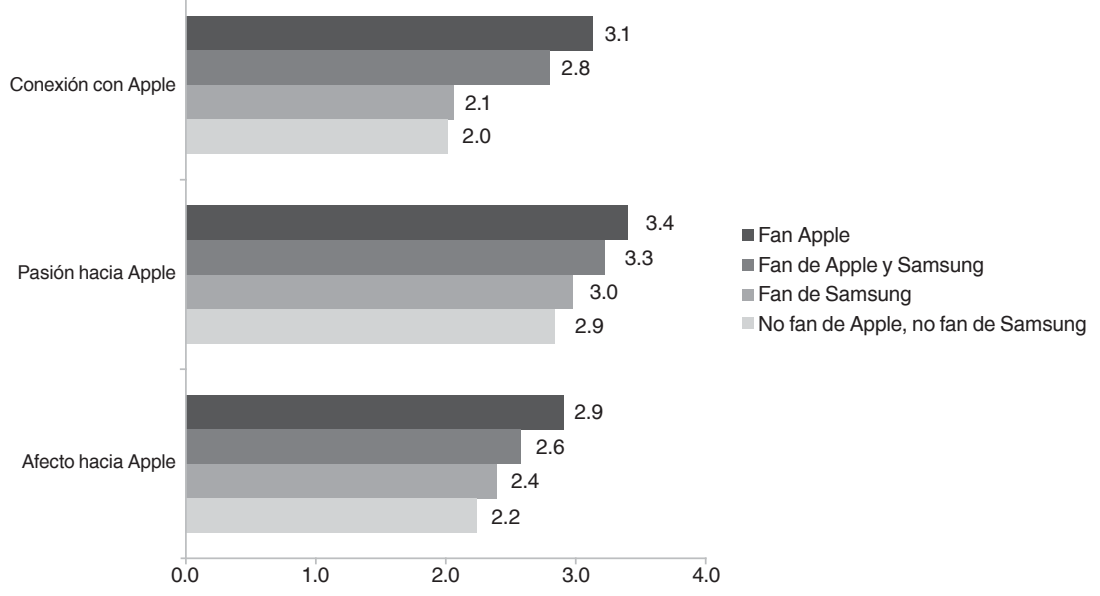

Fuente: Elaboración propia 
Gráfico 6. Valoración de las tres dimensiones de la marca Samsung para los cuatro grupos.

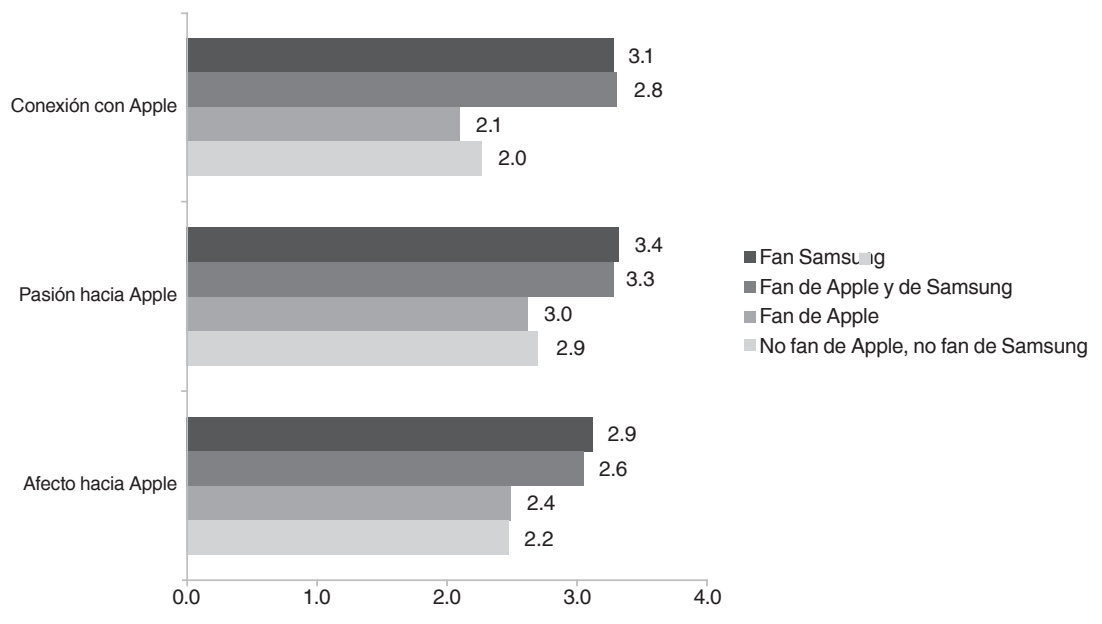

Fuente: Elaboración propia

\section{Conclusiones}

Los resultados del estudio trascienden el caso particular de las marcas Apple y Samsung al poner de manifiesto el papel del vínculo emocional hacia una marca en la relación que establecen los consumidores con dicha marca en el espacio determinado por las redes sociales.

El primer objetivo buscaba conocer el alcance de los fans de la marca Apple en el universo de las marcas de tecnología y telefonía entre los jóvenes. El 31\% de los participantes en el estudio declararon de manera espontánea ser fan de la marca Apple. Este es un porcentaje muy alto que pone de manifiesto el nivel de notoriedad que posee la marca.

El segundo objetivo pretendía averiguar la identificación de los atributos emocionales que se asocian a la marca Apple y si el vínculo emocional con dicha marca era superior a su rival Samsung como se ha evidenciado a lo largo del estudio. Por lo que se podría considerar que el vínculo emocional que se construye entre esta marca y el consumidor joven le inclina hacia la elección y preferencia de esta marca frente a otras.

Respecto al tercer objetivo, el aspecto más relevante del trabajo es que el vínculo afectivo es mayor entre los seguidores de la actividad de la marca en las redes sociales en las tres dimensiones: afecto, pasión y conexión con la marca. Además, este fenómeno también se produce en la marca Samsung, siendo el vínculo afectivo hacia la marca un elemento diferenciador entre los seguidores de la misma. Por lo tanto, se corrobora que el hecho de ser seguidor de una marca implica que se tiene más apego a ella.

Por lo tanto, en relación con las hipótesis planteadas en el estudio se confirma que:

a) la marca Apple genera mayor vínculo emocional que su competidora Samsung, coincidiendo con los valores 
aspiracionales que transmite la marca a través de su comunicación.

b) los seguidores de la marca Apple en las redes sociales manifiestan mayor vínculo emocional con la marca que los no seguidores.

c) los seguidores de la marca Samsung en redes sociales muestran mayor vínculo emocional con la marca que los que no lo son; por lo tanto se puede hablar del papel del vínculo emocional con la marca a la hora de iniciar la interacción en las redes sociales.

En otro orden de cosas, los resultados obtenidos nos permiten concluir, en líneas generales, que la adaptación de la escala del modelo de tres factores de Thomson, MacInnis, y Park (2005) arroja índices psicométricos satisfactorios cuando es aplicado a estas dos marcas por lo tanto se podría extender su aplicación para medir el vínculo afectivo a otras marcas del mercado.

\section{Bibliografía}

Ambler, T., \& Burne, T. (1999). The impact of affect on memory of advertising. Journal of Advertising Research, 39, 25-34.

Ambler, T. (2000). Persuasion, pride and prejudice: how ads work. International Journal of Advertising, 19(3), 299-315.

Arnold, D. (1992). Cómo gestionar una marca. Barcelona: Parramón.

Barry, T. E., \& Howard, D. J. (1990). A review and critique of the hierarchy of effects in advertising. International Journal of Advertising, 9(2), 121-135.

Benavides Delgado, J. (2014). Un nuevo enfoque para la comprensión de la marca y la comunicación de las empresas. En Sala, I. \& Mira, E. (2014) (coord.). Propectiva y tendencias para la comunicación del siglo XXI. CEU Ediciones: Madrid, pp. 515-535.

Churchill, G. A., JR. (1979). Marketing research: Methodological foundations. Hinsdale, IL: The Dryden Press

Gutiérrez, A. M. (2002). De lo que es capaz de conseguir la publicidad con las marcas. Investigación y Marketing, 75, pp.21-27.

IAB (2017). Estudio anual de redes sociales 2017. Disponible en https://www.slideshare.net/elogia/iab-estudio-anual-redes-sociales-2017.

Krugman, H. E. (1965). The impact of television advertising: Learning without involvement. Public opinion quarterly, 29(3), 349-356.

Lavidge, R. J. \& Steiner, G. A. (2000). A model for predictive measurements of advertising effectiveness. Advertising \& Society Review, 1(1).

López Vázquez, B. (2007). Publicidad emocional. Estrategias creativas. Madrid: ESIC.

Martín Requero, M. I. \& Cruz Alvarado López, M. C. (2007). Nuevas tendencias en la publicidad del siglo XXI. Sevilla: Comunicación Social.

Petty, R. E. \& Cacioppo, J.T. (1986). The elaboration likelihood model of persuasion. New York: Academic Press.

Roberts, K. (2005). Lovemarks: The Future Beyond Brands. New York: Power House Books.

Strong, E. K. (1925). The Psychology of Selling. Nueva York: MacGraw Hill. 


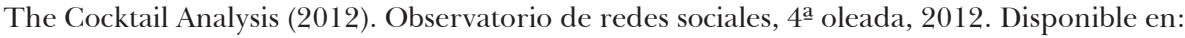
http//www.slideshare.net/TCAnalysis/4-oleada-observatorio-de-redes-sociales/download.

The Cocktail Analysis (2016). Observatorio de redes sociales, 8aㅡ oleada, 2016. Disponible en: http://tcanalysis.com/blog/posts/viii-observatorio-de-redes-sociales.

Thomson, M., MacInnis, D. J. \& Whan Park, C. (2005). The ties that bind: Measuring the strength of consumers' emotional attachments to brands. Journal of consumer psychology, 15(1), 77-91.

TNS (2012): Digital life 2012. Disponible en http://www.tnsglobal.com/sites/tnsglobal.es/files/ index_jul_13_web.html.

Vakratsas, D., \& Ambler, T. (1999). How advertising works: what do we really know?. The Journal of Marketing, 26-43.

Vilaseca, B. (17/9/2006). En la mente del consumidor, El País. Recuperado de http://elpais. com/diario/2006/09/17/negocio/1158500854_850215.html.

Zeitlin, D. M. \& Westwood, R. A. (1986). Measuring Emotional Reponse. Journal of Advertising Research, pp. 34-44. 